\title{
Bateson Information Revisited: A New Paradigm ${ }^{\dagger}$
}

\author{
Jaime F. Cárdenas-García 1,* and Timothy Ireland ${ }^{2}$ \\ 1 Department of Mechanical Engineering, University of Maryland, Baltimore County, 1000 Hilltop Circle, \\ Baltimore, MD 21250, USA \\ 2 Kent School of Architecture, University of Kent, Marlowe Building, Canterbury CT2 7NR, UK; \\ t.ireland@kent.ac.uk \\ * Correspondence: jfcg@umbc.edu; Tel.: +1-240-498-7556 \\ + Conference Theoretical Information Studies (TIS), Berkeley, CA, USA, 2-6 June 2019.
}

Published: 11 May 2020

\begin{abstract}
The goal of this work is to explain a novel information paradigm claiming that all information results from a process, intrinsic to living beings, of self-production; a sensory commensurable, self-referential feedback process immanent to Bateson's difference that makes a difference. To highlight and illustrate this fundamental process, a simulation based on one-parameter feedback is presented. It simulates a homeorhetic process, innate to organisms, illustrating a self-referenced, autonomous system. The illustrated recursive process is sufficiently generic to be the only basis for information in nature: from the single cell, to multi-cellular organisms, to consideration of all types of natural and non-natural phenomena, including tools and artificial constructions.
\end{abstract}

Keywords: Gregory Bateson; homeorhesis; one-parameter feedback; self-reference; autonomy; sensory commensurable; self-production of information

\section{Introduction}

Gregory Bateson is well known for defining information as a difference that makes a difference [1] (p. 453). Such a succinct and deceptively simple definition is certainly subject to possible misinterpretation. One such misinterpretation might involve suggesting that it is a circuitous, self-referential play on the word difference which leads nowhere, since differences seem to be ubiquitous. In this general misinterpretation, the missing important detail is that the organism-in-its-environment, as a representative of all living beings, is at the centre of assessing differences. For us humans, we start our process of distinguishing differences at the time of our conception as living beings. When a single human cell becomes two cells, a further division leads to four cells and so on. Until the emergence of the child from the womb, to begin an additional gestation period out of the womb. This cumulative composite of cells is certain to detect spatial/temporal differences that allows this process to become effective. Without delving into all of the biological complexity that probably plays a role, at some point our five primary senses (touch, sight, hearing, smell and taste) come on line perpetually. Our senses function continuously, consciously or unconsciously, to detect spatial and/or temporal differences in our dynamic environment. In the initial period of gestation out of the womb, our senses help us to sound the alarm to be nurtured when hungry and held close for warmth.

To begin the journey of determining differences using our five primary senses, it is important to note that our senses deal with commensurable quantities/qualities. For example, the sense of touch (whose multidimensional structure includes mechanoreceptors, thermoreceptors, nocireceptors, proprioceptors) might be, for simplicity, arbitrarily ascribed as being sensitive only to pressure. In that limited role, our sense of touch is able to keep track of all pressure sensations that come into its 
sphere of action. As might be imagined, from one instant of time to the next, pressure sensations are felt by the human in question and become part of her experience. This is how quantitatively and unambiguously 'a (pressure) difference' becomes qualitatively 'a (pressure) difference that makes a difference'. In a similar way, the other dimensions of the sense of touch contribute with their own unique quantitative/qualitative characteristics and thus, contribute to a multidimensional sensory experience that consists of temporal/spatial differences. Implicit to this conception of information, applicable to all living beings, is that all information is self-produced by all living beings. A corollary is that there is no information in the environment, except for information produced by living beings.

To discuss the process of self-produced information by all living beings and its implications, this paper is divided into three sections. First, a homeorhetic feedback simulation is used to gain a perspective on this approach to information. Second, a brief discussion puts all the pieces together. Finally, the findings of the paper are summarized and pertinent conclusions are presented.

\section{A One-Parameter Feedback Simulation of Homeorhesis}

Norbert Wiener tautologically states that 'Information is information, not matter or energy. No materialism, which does not admit this, can survive at the present day' [2] (p. 132). This is how Wiener accredited that information is a most pervasive and unique element. Even proposing that information is a third fundamental quantity of the Universe, which is a widely held perspective [3-8]. The definition of Bateson information as a difference that makes a difference challenges this conception of information. What is widely acknowledged is that matter and/or energy are the only fundamental quantities of the Universe. Further, matter and/or energy are in motion above a temperature of zero degrees absolute. All living beings are able to detect the spatial and temporal dynamics of their environment. More practically, what is required is that living beings be capable of comparing two spatial/temporal instances so as to discern a difference. Thus, there is no need for declaring information as a third quantity of the Universe.

A comparison between two (or more) spatial/temporal instances, at the most elementary level, can be implemented using a one-parameter feedback loop simulation utilizing a sensor and a comparator. The comparator requires two commensurable inputs: a reference parameter that is set, internally or externally to the system, and a detected parameter supplied by a sensor. This arrangement implies the notion of constitutive absence [9].

To exemplify this approach, we examine the one-parameter feedback simulation of homeorhetic reflex-actions in an organism. Homeorhesis indicates system dynamics with different transitions to multi-stable trajectories. Waddington suggested that, in biological systems, homeorhesis (stability of dynamics rather than stability of states) instead of homeostasis prevails. In other words, what 'is being held constant is not a single parameter but is a time-extended course of change, that is to say, a trajectory' [10] (p. 12). An organism may be considered, for the most part, as a collection of reflex-actions, i.e., involuntary and nearly instantaneous movements in response to a stimulus. An important assumption here is that reflex-actions are phylogenetic behaviour that can be considered as the first layer of homeorhesis [11].

Figure 1 shows a feedback simulation of a prototypical organism-in-its-environment depicting cyclic self-referenced reflex-action operations to keep homeorhetic trajectories. Note that the two elements portray the organism as subsumed in the environment. In Figure 1, consider the beginning of an organism-in-its-environment cyclic interactions as the detection of environmental noise by the senses of the organism. This is the only window that the organism has to access its environment. Environmental noise is particular to each individual, since each individual has a particular set of senses that are attuned to its phylogenetic and ontogenetic development within a specified environment. The primary motivation of the organism in sensing the noisy environment that may resemble white noise, is to maintain its individuation and homeorhetic trajectories in epigenetic landscapes due to dynamic openness [10]. For example, the organism needs to satisfy its energy needs and is tuned to particular cues in the white noise that leads it to satisfy them [12-14]. 


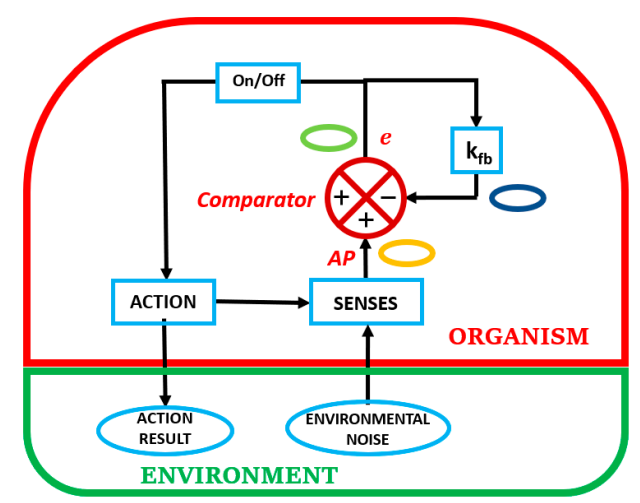

Figure 1. Simulation of a homeorhetic organism-in-its-environment.

The portrayal in Figure 1 defines the fundamental condition of the organism, as it exists embedded in its environment. There are two essential connections with the environment. One is shown as a single sense element that is the intermediary between the external environment and the internal milieu of the organism. This single sense element represents a microcosm of reality, since a multi-celled organism is composed of millions of these sense elements that define each particular sense organ in the body. The other connection is the capacity of the organism to physically impact the environment, either directly or by other means. Our intent in what follows is to concentrate on the sensorial side of this dichotomy, as the single sense element is the only means that an organism has to ascertain the reality of the external environment to successfully engage it.

The transduction role of the single sense element changes the physical (touch, sound, light) or chemical signature (smell, taste) to a corresponding electrical signal or action potential (AP). It is this $\mathrm{AP}$, irrespective of origin, that is used by the human organism, either locally or centrally, to generate information: a difference that makes a difference. This process is akin to the Principle of Undifferentiated Encoding, which states that, "The response of a nerve cell does not encode the physical nature of the agents that caused its response. Encoded is only "how much" at this point on my body, but not "what"' [15] (p. 4). Except that the AP that needs to be used, either locally or centrally, is representative of self-produced information. As a result, we suggest that the above Principle may be alternatively defined, being more encompassing, in terms of self-produced information.

Examining Figure 1 shows the Comparator as having a feedback circuit that incorporates a quantity $k_{f b}$ to modify the error, $e$. The feedback signal independently modifies the incoming sensory $A P$ by subtracting a factor $e k_{f b}$, The quantity, $k_{f b}$, is a function of the needs of the organism.

An equation that can be obtained from looking at the comparator, where $e$ is the error and AP is the Action Potential, yields a relationship between input and output given by,

$$
\frac{e}{A P}=\frac{1}{\left(1+k_{f b}\right)}
$$

The quantity $k_{f b}$ may be regarded as a function of difference, $e$, of time, and other factors internal and external to the organism. The relationship between input and output is capable of many fluctuations, to accommodate multifaceted behaviour.

On the left side of composite Figure 2, we find a plot of the output over the input, i.e., e/AP. In this particular case, a constant value of $A P=1$ is used, and $k_{f b}$ varies from 0.1 to 1.0 in increments of 0.1 resulting in 10 curves generated at 10 time-steps of unspecified length. Note that the colour coding in the two images on the right side is used to show how the calculation of $e / A P$ is performed. The curves in the graph show the versatility of the reflex-action depending on the value of $k_{f b}$. The curve for a value of $k_{f b}=1.0$ envelops all the other curves as it oscillates between the values of 0 and 1 over time, implying continuous triggering of the reflex-action. All successive curves show an oscillatory reduction over time. 
Referring again to Figure 2 (top right insert), note that an On/Off trigger switch is present. This trigger switch will remain On for difference, error or information, e, values above a certain reference value (not to be confused with the reference parameter $\left(e k_{f b}\right)$ ), but will remain Off below that same reference value. Looking at the graph in Figure 2, if an arbitrary trigger reference value is set to a value of $e=0.85$, the reflex-action will trigger once for all values of $k_{f b}$, but will trigger four additional times for a value of $k_{f b}=1.0$; one additional time for a value of $k_{f b}=0.9$; and, will remain triggered continuously for a value of $k_{f b}=0.1$.
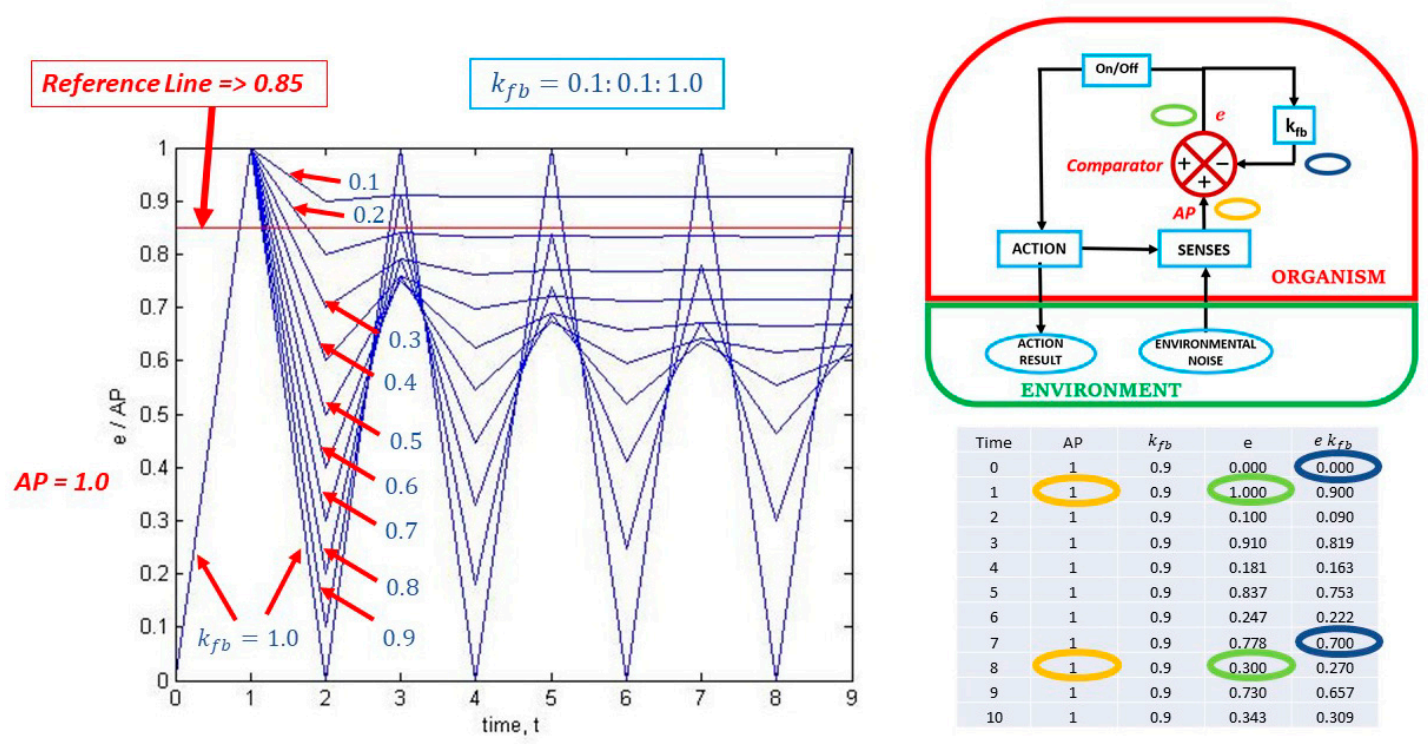

Figure 2. The effect of changing $k_{f b}$ on information self-production.

In short, a four-step conceptualization of how this feedback simulation of the organism consisting of a one-parameter Sensor and Comparator works, is as follows:

- A comparator is set to a reference parameter, defined by $e k_{f b}$;

- The sensor (one of the main senses) distinguishes the value of the detected parameter;

- The comparator obtains the difference or error between the detected parameter and the reference parameter;

- The detected difference or error is the information needed to allow the actuation of a reflex-action when it exceeds an organism determined reference value.

In summary, the process of self-produced information is shown to be a self-referenced process that is common to all living beings. The implication is that information only exists in nature as the result of self-production.

\section{Discussion}

The focus of this paper is to propose the process of self-referenced self-production of information by living beings as the source of all information, i.e., Bateson's difference that makes a difference.

To highlight the general nature of information self-production, a simulation based on one-parameter feedback is introduced: a homeorhetic process inherent to biological systems. This serves to illustrate the generic nature of information as a difference that makes a difference. The nature of the difference is fundamental to deal with quantities/qualities that are commensurable.

In the theoretical homeorhetic one-parameter feedback loop simulation, information is obtained using an action potential $(A P)$ as a basis for self-production of information. The driving $A P$ originates from selectively filtered environmental noise, which feedbacks into the comparator to make a comparison using a single sensor. It is then possible to obtain an error or difference or information from commensurable quantities/qualities to cause a specified reflex-action. 


\section{Summary and Conclusions}

One of the most vexing aspects of information is the inability to define it to include syntactic/quantitative and semantic/qualitative elements. The definition by Gregory Bateson of information as a difference that makes a difference is shown to have this ability [16]. Additionally, a sensory commensurable, self-referential feedback process may be shown to be inherent to Bateson's conceptualization of information. Further, the process of information self-production is fundamental to the conception of information. The unavoidable implication is that there is no information outside of that generated by living beings through the process of interaction with their environment.

What is suggested above contradicts the common notions of information in biology, where information is considered to be external to the organism and seems to have a purely objective, standalone existence, able to be processed, stored and transferred. In short, self-produced information is a recursive process that exists in all biological systems and is postulated as the basis to understand information in any biological phenomena: from the single cell, to multi-cellular organisms, to consideration of all types of natural and non-natural phenomena, including tools and artificial constructions.

Author Contributions: Conceptualization, J.F.C.-G. and T.I.; methodology, J.F.C.-G.; writing-original draft preparation, J.F.C.-G.; writing-review and editing, J.F.C.-G. and T.I. All authors have read and agreed to the published version of the manuscript.

Funding: This research received no external funding.

Acknowledgments: The authors would like to acknowledge the encouragement of Mark Burgin.

Conflicts of Interest: The authors declare no conflict of interest.

\section{References}

1. Bateson, G. Steps to an Ecology of Mind. In Collected Essays in Anthropology, Psychiatry, Evolution, and Epistemology, Chandler Publications for Health Sciences; Ballantine Books: New York, NY, USA, 1978.

2. Wiener, N. Cybernetics: Or Control and Communication in the Animal and the Machine, 2nd ed.; MIT Press: Cambridge, MA, USA, 1961; First Published in 1948.

3. Burgin, M. Theory of Information-Fundamentality, Diversity and Unification; World Scientific Series in Information Studies; World Scientific Publishing Co. Pte. Ltd.: Singapore, 2010; Volume 1.

4. Floridi, L. Information: A Very Short Introduction; Oxford University Press: Oxford, UK, 2010.

5. Lloyd, S. Programming the Universe; Alfred A. Knopf: New York, NY, USA, 2006.

6. Stonier, T. Information and Meaning-An Evolutionary Perspective; Springer: Berlin/Heidelberg, Germany; New York, NY, USA, 1997.

7. Vedral, V. Decoding Reality - The Universe as Quantum Information; Oxford University Press: Oxford, UK, 2010.

8. Yockey, H.P. Information Theory, Evolution, and the Origin of Life; Cambridge University Press: Cambridge, UK, 2005.

9. Deacon, T.W. Emergence: The Hole at the Wheel's Hub. In The Re-Emergence of Emergence: The Emergentist Hypothesis from Science to Religion; Philip, C., Paul, D., Eds.; Oxford University Press: Oxford, UK, 2008; pp. $111-150$.

10. Waddington, C.H. Towards a Theoretical Biology: An International Union of Biological Sciences Symposium; Nature Publishing Group: Edinburgh, UK, 1968.

11. Huntingford, F. The Study of Animal Behaviour; Chapman and Hall: London, UK; New York, NY, USA, 1984.

12. Cárdenas-García, J.F. Distributed Cognition: An Ectoderm-Centric Perspective. Biosemiotics 2013, 6, 337-350.

13. Cárdenas-García, J.F.; Timothy, I. Human Distributed Cognition from an Organism-in-Its-Environment Perspective. Biosemiotics 2017, 10, 265-278.

14. Cárdenas-García, J.F.; Timothy, I. The Fundamental Problem of the Science of Information. Biosemiotics 2019, 12, 213-244. 
15. Von Foerster, H. Understanding Understanding: Essays on Cybernetics and Cognition; Springer: Berlin/Heidelberg, Germany, 2003.

16. Cárdenas-García, J.F.; Diego, R.C.; de Mesa, B.S. Object Discernment by “a Difference Which Makes a Difference". Biosemiotics 2018, 11, 27-40.

(C) 2020 by the authors. Licensee MDPI, Basel, Switzerland. This article is an open access article distributed under the terms and conditions of the Creative Commons Attribution (CC BY) license (http://creativecommons.org/licenses/by/4.0/). 